\title{
Computing generating functions of ordered partitions with the transfer-matrix method
}

\author{
Masao Ishikawa ${ }^{1}$, Anisse Kasraoui ${ }^{2}$ and Jiang Zeng ${ }^{2}$ \\ ${ }^{1}$ Faculty of Education, Tottori University, Koyama, Tottori, Japan \\ ${ }^{2}$ Institut Camille Jordan, Université Claude Bernard (Lyon I), F-69622 Villeurbanne Cedex, France
}

An ordered partition of $[n]:=\{1,2, \ldots, n\}$ is a sequence of disjoint and nonempty subsets, called blocks, whose union is $[n]$. The aim of this paper is to compute some generating functions of ordered partitions by the transfer-matrix method. In particular, we prove several conjectures of Steingrímsson, which assert that the generating function of some statistics of ordered partitions give rise to a natural $q$-analogue of $k ! S(n, k)$, where $S(n, k)$ is the Stirling number of the second kind.

Keywords: Ordered partitions, Euler-Mahonian statistics, $q$-Stirling numbers of second kind, transfer-matrix method, determinants.

\section{Contents}

1 Introduction 193

1.1 Background . . . . . . . . . . . . . . . . . . . . . . . . . . . . . . 193

1.2 Definitions. . . . . . . . . . . . . . . . . . . . . . . . . . . . . . . . . . . 194

1.3 Main results . . . . . . . . . . . . . . . . . . . . . . . . . . . . . . . . 196

\begin{tabular}{|lll}
2 & Ordered partitions and walks in digraphs & 197
\end{tabular}

$2.1 \quad$ Encoding ordered partitions by walks $\ldots \ldots \ldots \ldots$. . . . . . . . . . . . 197

2.2 Generating functions of walks $\ldots \ldots \ldots \ldots \ldots \ldots$

3 Determinantal computations $\quad 200$

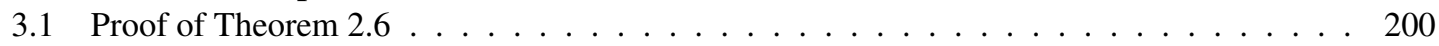

3.2 Proof of Theorem $2.7 \ldots \ldots \ldots \ldots \ldots$

\section{Introduction}

\subsection{Background}

Definition 1.1 A partition $\pi_{0}$ of $[n]=\{1,2, \ldots, n\}$ is a collection of disjoint and nonempty subsets, called blocks, whose union is $[n]$. By convention, we write $\pi_{0}=B_{1}-B_{2}-\cdots-B_{k}$, where the blocks $B_{i}$ are arranged in increasing order of their minimal elements and within each block the elements are arranged in increasing order.

For instance, $\pi_{0}=1-246-35-78$ is a partition of [8] with 4 blocks.

Let $\mathcal{P}_{n}^{k}$ be the set of partitions of $[n]$ with $k$ blocks. In the present paper we study statistics on ordered partitions, that is, partitions where the blocks are ordered arbitrarily.

Definition 1.2 An ordered partition $\pi$ of $[n]$ with $k$ blocks is just a rearrangement of blocks of a partition in $\mathcal{P}_{n}^{k}$, that is there exist $\pi_{0}=B_{1}-B_{2}-\cdots-B_{k} \in \mathcal{P}_{n}^{k}$ and $\sigma$ a permutation of $[k]$ such that $\pi=$ $B_{\sigma(1)}-B_{\sigma(2)}-\cdots-B_{\sigma(k)}$. We will say that $\sigma$ is the permutation induced by $\pi$ and set $\sigma=\operatorname{perm}(\pi)$.

For instance, $\pi=35-246-1-78$ is an ordered partition of [8] with 4 blocks and we have $\operatorname{perm}(\pi)=$ 3214.Let $\mathcal{O} \mathcal{P}_{n}^{k}$ be the set of ordered partitions of $[n]$ into $k$ blocks. It is well known that the cardinality of $\mathcal{P}_{n}^{k}$ is the Stirling number of the second kind $S(n, k)$. It follows that $k ! S(n, k)$ counts the ordered partitions of $[n]$ with $k$ blocks. 
Let $\sigma=\sigma(1) \sigma(2) \ldots \sigma(n)$ be a permutation of $[n]$, the integer $i \in[n-1]$ is called a descent of $\sigma$ if $\sigma(i)>\sigma(i+1)$. The Eulerian number $A(n, k)$ counts permutations of $[n]$ with $k$ descents. There is a basic identity relating the $S(n, k)$ 's and the $A(n, k)$ 's, namely

$$
k ! S(n, k)=\sum_{m=1}^{k}\left(\begin{array}{c}
n-m \\
n-k
\end{array}\right)\left\langle\begin{array}{c}
n \\
m-1
\end{array}\right\rangle,
$$

which is easily proved combinatorially (for instance, see [10] ).

In the present paper we will study some statistics originated from a $q$-analogue of (1) by means of the $q$-Eulerian numbers and $q$-Stirling numbers of the second kind, which were introduced by Carlitz [1, 2]. We need more definitions and notations.

Define the $p, q$-integer $[n]_{p, q}=\frac{p^{n}-q^{n}}{p-q}$, the $p, q$-factorial $[n]_{p, q} !=[1]_{p, q}[2]_{p, q} \cdots[n]_{p, q}$ and the $p, q$ binomial coefficients

$$
\left[\begin{array}{c}
n \\
k
\end{array}\right]_{p, q}=\frac{[n]_{p, q} !}{[k]_{p, q} ![n-k]_{p, q} !} \quad n \geq k \geq 0 .
$$

If $p=1$, we shall write $[n]_{q},[n]_{q}$ ! and $\left[\begin{array}{l}n \\ k\end{array}\right]_{q}$ for $[n]_{1, q},[n]_{1, q}$ ! and $\left[\begin{array}{l}n \\ k\end{array}\right]_{1, q}$ respectively.

The $q$-Eulerian numbers $A_{q}(n, k)(n \geq k \geq 0)$ are defined by

$$
A_{q}(n, k)=q^{k}[n-k]_{q}\left\langle\begin{array}{l}
n-1 \\
k-1
\end{array}\right\rangle_{q}+[k+1]_{q}\left\langle\begin{array}{c}
n-1 \\
k
\end{array}\right\rangle_{q} .
$$

Let $\sigma$ be a permutation of $[n]$, define the statistic maj $\sigma=\sum_{i} i$ where the summation is over all descents of $\sigma$. Then

$$
A_{q}(n, k)=\sum_{\sigma} q^{\operatorname{maj} \sigma},
$$

where the summation is over all permutations of $[n]$ with $k$ descents.

The $q$-Stirling numbers $S_{q}(n, k)$ of the second kind are defined by:

$$
S_{q}(n, k)=q^{k-1} S_{q}(n-1, k-1)+[k]_{q} S_{q}(n-1, k) \quad(n \geq k \geq 0),
$$

where $S_{q}(n, k)=\delta_{n k}$ if $n=0$ or $k=0$.

A $q$-analogue of (1) has been found in [15] as follows:

$$
[k]_{q} ! S_{q}(n, k)=\sum_{m=1}^{k} q^{k(k-m)}\left[\begin{array}{c}
n-m \\
n-k
\end{array}\right]_{q}\left\langle\begin{array}{c}
n \\
m-1
\end{array}\right\rangle_{q} .
$$

There has been a considerable amount of recent interest in properties and combinatorial interpretations of the $q$-Stirling numbers and related numbers (see e.g. [1, 2, 4, 5, 6, 7, 8, 10, 11, 12, 13]). In the aim to give a combinatorial proof of [3], Steingrímsson [10] introduced the following definition.

Definition 1.3 A statistic Stat on $\mathcal{O P}_{n}^{k}$ is called Euler-Mahonian if its generating function is equal to $[k]_{q} ! S_{q}(n, k)$, i.e.,

$$
\sum_{\pi \in \mathcal{O} \mathcal{P}_{n}^{k}} q^{\text {Stat } \pi}=[k]_{q} ! S_{q}(n, k) .
$$

Steingrímsson [10] has found a few of Euler-Mahonian statistics and has introduced new statistics on ordered partitions. Moreover, he has conjectured that all these new statistics are Euler-Mahonian. Wachs [11] has also obtained some Euler-Mahonian statistics on ordered partitions.

\subsection{Definitions}

Given a partition $\pi$ in $\mathcal{O} \mathcal{P}_{n}^{k}$, the elements of $[n]$ are divided into four classes:

- singletons: elements of the singleton blocks;

- openers: smallest elements of the non singleton blocks;

- closers: largest elements of the non singleton blocks;

- transients: all other elements, i.e., non extremal elements of non singleton blocks. 
The sets of openers, closers, singletons and transients of $\pi$ will be denoted by $\mathcal{O}(\pi), \mathcal{C}(\pi), \mathcal{S}(\pi)$ and $\mathcal{T}(\pi)$, respectively. The 4-tuple $(\mathcal{O}(\pi), \mathcal{C}(\pi), \mathcal{S}(\pi), \mathcal{T}(\pi))$ is called the type of $\pi$. For instance, if $\pi=$ $35-246-1-78$, then

$$
\mathcal{O}(\pi)=\{2,3,7\}, \quad \mathcal{C}(\pi)=\{5,6,8\}, \quad \mathcal{S}(\pi)=\{1\} \quad \text { and } \quad \mathcal{T}(\pi)=\{4\}
$$

Definition 1.4 Let $\sigma$ be a permutation of $[n]$; the pair $(i, j)$ is an inversion if $1 \leq i<j \leq n$ and $\sigma(i)>$ $\sigma(j)$. Let inv $\sigma$ be the number of inversions in $\sigma$. We also define the number of inversions of an ordered partition $\pi$, inv $\pi$, by inv $\pi=\operatorname{inv}($ perm $(\pi))$. For a partition $\pi$ in $\mathcal{O} \mathcal{P}^{k}$, we also set $\operatorname{cinv} \pi=\left(\begin{array}{c}\mathrm{k} \\ 2\end{array}\right)-\operatorname{inv} \pi$.

Let $\pi=B_{1}-B_{2}-\cdots-B_{k}$ be a partition in $\mathcal{O} \mathcal{P}_{n}^{k}$. We define a partial order on blocks $B_{i}$ 's as follows : $B_{i}>B_{j}$ if all the letters of $B_{i}$ are greater than those of $B_{j}$; in other words, if the opener of $B_{i}$ is greater than the closer of $B_{j}$.

Definition 1.5 A block inversion in $\pi$ is a pair $(i, j)$ such that $i<j$ and $B_{i}>B_{j}$. We denote by bInv $\pi$ the number of block inversions in $\pi$. We also set $\mathrm{cbInv}=\left(\begin{array}{c}\mathrm{k} \\ 2\end{array}\right)-$ bInv.

Let $w_{i}$ be the index of the block (counting from the left) containing $i$, namely the integer $j$ such that $i \in B_{j}$. Following Steingrímsson [10], we define for $1 \leq i \leq n$ ten coordinate statistics on $\pi \in \mathcal{O} \mathcal{P}_{n}^{k}$ :

$$
\begin{aligned}
\operatorname{ros}_{\mathrm{i}}(\pi) & =\#\left\{j \in(\mathcal{O} \cup \mathcal{S})(\pi) \mid j<i, w_{j}>w_{i}\right\}, \\
\operatorname{rob}_{\mathrm{i}}(\pi) & =\#\left\{j \in(\mathcal{O} \cup \mathcal{S})(\pi) \mid j>i, w_{j}>w_{i}\right\}, \\
\operatorname{rcs}_{\mathrm{i}}(\pi) & =\#\left\{j \in(\mathcal{C} \cup \mathcal{S})(\pi) \mid j<i, w_{j}>w_{i}\right\}, \\
\operatorname{rbb}_{\mathrm{i}}(\pi) & =\#\left\{j \in(\mathcal{C} \cup \mathcal{S})(\pi) \mid j>i, w_{j}>w_{i}\right\}, \\
\operatorname{los}_{\mathrm{i}}(\pi) & =\#\left\{j \in(\mathcal{O} \cup \mathcal{S})(\pi) \mid j<i, w_{j}<w_{i}\right\}, \\
\operatorname{lob}_{\mathrm{i}}(\pi) & =\#\left\{j \in(\mathcal{O} \cup \mathcal{S})(\pi) \mid j>i, w_{j}<w_{i}\right\}, \\
\operatorname{lcs}_{\mathrm{i}}(\pi) & =\#\left\{j \in(\mathcal{C} \cup \mathcal{S})(\pi) \mid j<i, w_{j}<w_{i}\right\}, \\
\operatorname{lcb}_{\mathrm{i}}(\pi) & =\#\left\{j \in(\mathcal{C} \cup \mathcal{S})(\pi) \mid j>i, w_{j}<w_{i}\right\},
\end{aligned}
$$

where $(\mathcal{O} \cup \mathcal{S})(\pi)=\mathcal{O}(\pi) \cup \mathcal{S}(\pi)$, and let $\operatorname{rsb}_{\mathrm{i}}(\pi)$ (resp. $\operatorname{lsb}_{\mathrm{i}}(\pi)$ ) be the number of blocks in $\pi$ to the right (resp. left) of the block containing $i$ such that the opener of $\mathrm{B}$ is smaller than $i$ and the closer of $\mathrm{B}$ is greater than $i$. Then define ros, rob, rcs, rcb, lob, los, lcs, lcb, lsb and rsb as the sum of their coordinate statistics, e.g.

$$
\operatorname{ros}=\sum_{\mathrm{i}} \operatorname{ros}_{\mathrm{i}}
$$

Remark 1.6 Note that ros is the abbreviation of "right, opener, smaller", while lsb is the abbreviation of "left, closer, bigger", etc.

More generally, if stat is one of the above ten statistics, we define $\operatorname{stat}(\mathcal{O} \cup \mathcal{S})$ by

$$
\operatorname{stat}(\mathcal{O} \cup \mathcal{S})(\pi)=\sum_{i \in(\mathcal{O} \cup \mathcal{S})(\pi)} \operatorname{stat}_{i}(\pi)
$$

In the same way, we define $\operatorname{stat}(\mathcal{T} \cup \mathcal{C}), \operatorname{stat}(\mathcal{C} \cup \mathcal{S})$, etc. The following results illustrate the above notions.

Proposition 1.7 The following functional identities hold on $\mathcal{O} \mathcal{P}^{k}$ :

$$
\mathrm{bInv}=\operatorname{rcs}(\mathcal{O} \cup \mathcal{S}), \quad \operatorname{inv}=\operatorname{ros}(\mathcal{O} \cup \mathcal{S}) \quad \text { and } \quad \operatorname{cinv}=\operatorname{los}(\mathcal{O} \cup \mathcal{S})
$$


For instance, we give the values of the coordinate statistics computed on the partition $\pi=68-5-$ $147-39-2$ :

$$
\begin{aligned}
& \pi=68-5-147-39-2 \\
& \operatorname{los}_{\mathrm{i}}: 00-0-002-13-1 \\
& \operatorname{ros}_{\mathrm{i}}: 44-3-022-11-0 \\
& \mathrm{lob}_{\mathrm{i}}: 00-1-220-20-3 \\
& \text { rob }_{\mathrm{i}}: 00-0-200-00-0 \\
& \operatorname{lcs}_{\mathrm{i}}: 00-0-001-03-0 \\
& \mathrm{rcs}_{\mathrm{i}}: 23-1-011-11-0 \\
& \mathrm{lcb}_{\mathrm{i}}: 00-1-221-30-4 \\
& \mathrm{rcb}_{\mathrm{i}}: 21-2-211-00-0 \\
& \mathrm{lsb}_{\mathrm{i}}: 00-0-001-10-1 \\
& \mathrm{rsb}_{\mathrm{i}}: 21-2-011-00-0
\end{aligned}
$$

Since $\{6,8\}>\{5\},\{6,8\}>\{2\},\{5\}>\{2\}$ and $\{3,9\}>\{2\}$, we have bInv $\pi=4$ and $\operatorname{cbInv} \pi=$ $\left(\begin{array}{l}5 \\ 2\end{array}\right)-4=6$. Moreover, $\operatorname{perm}(\pi)=54132, \operatorname{thus} \operatorname{inv}(\pi)=8$ and $\operatorname{cinv}(\pi)=\left(\begin{array}{l}5 \\ 2\end{array}\right)-8=2$.

Inspired by the statistic mak on the permutations due to Foata \& Zeilberger [3] , Steingrímsson introduced its analogous on $\mathcal{O} \mathcal{P}_{n}^{k}$ as follows:

$$
\begin{aligned}
\text { mak } & =\operatorname{ros}+\mathrm{lcs}, \\
\operatorname{lmak} & =n(k-1)-[\mathrm{los}+\mathrm{rcs}] \\
\mathrm{mak}^{\prime} & =\mathrm{lob}+\mathrm{rcb}, \\
\operatorname{lmak}^{\prime} & =n(k-1)-[\mathrm{lcb}+\mathrm{rob}] .
\end{aligned}
$$

The following proposition permits to reduce the conjecture 12 in [10] almost by half. This result was first proved in [4].

Proposition 1.8 On $\mathcal{O P}{ }_{n}^{k}$ the following functional identities hold:

$$
\text { mak }=\operatorname{lmak}^{\prime} \text { and } \operatorname{mak}^{\prime}=\text { lmak. }
$$

Define also the statistic cinvLSB on $\mathcal{O P}^{k}$ by

$$
\operatorname{cinvLSB}:=\mathrm{lsb}+\mathrm{cbInv}+\left(\begin{array}{l}
\mathrm{k} \\
2
\end{array}\right)
$$

\subsection{Main results}

Consider the following two generating functions of ordered partitions with $k \geq 0$ blocks:

$$
\begin{aligned}
\phi_{k}(a ; x, y, t, u) & :=\sum_{\pi \in \mathcal{O P}^{k}} x^{(\mathrm{mak}+\mathrm{bInv}) \pi} y^{\operatorname{cinvLSB} \pi} t^{\mathrm{inv} \pi} u^{\operatorname{cinv} \pi} a^{|\pi|} \\
\varphi_{k}(a ; z, t, u) & :=\sum_{\pi \in \mathcal{O P}^{k}} z^{(\mathrm{lmak}+\mathrm{bInv}) \pi} t^{\operatorname{inv} \pi} u^{\operatorname{cinv} \pi} a^{|\pi|}
\end{aligned}
$$

where $|\pi|=n$ if $\pi$ is a partition of $[n]$. The following Theorem is the main result of this paper.

Theorem 1.9 We have

$$
\begin{aligned}
\phi_{k}(a ; x, y, t, u) & =\frac{a^{k}(x y)^{\left(\begin{array}{c}
k \\
2
\end{array}\right)}[k]_{t x, u y} !}{\prod_{i=1}^{k}\left(1-a[i]_{x, y}\right)}, \\
\varphi_{k}(a ; z, t, u) & =\frac{a^{k} z^{\left(\begin{array}{c}
k \\
2
\end{array}\right)}[k]_{t z, u} !}{\prod_{i=1}^{k}\left(1-a[i]_{z}\right)} .
\end{aligned}
$$

The above theorem infers results on Euler-Mahonian statistics on ordered partitions. Indeed, it follows directly from (2) that

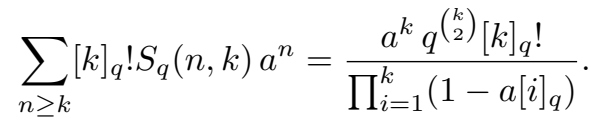

Then, by appropriate specializations in $(6)$ and $(7)$, we obtain the following result conjectured by Steingrímsson [10, conj.12 and 13]. 
Theorem 1.10 The following statistics are Euler-Mahonian on $\mathcal{O} \mathcal{P}_{n}^{k}$ :

$$
\text { mak }+ \text { bInv, lmak + bInv, cinvLSB. }
$$

In other words, the generating functions of the above statistics over $\mathcal{O} \mathcal{P}_{n}^{k}$ are all equal to $[k]_{q} ! S_{q}(n, k)$.

Others specializations give rise to new Euler-Mahonian statistics.

Theorem 1.11 The following statistics are Euler-Mahonian on $\mathcal{O P}_{n}^{k}$ :

$$
\text { mak }+ \text { bInv }-(\text { inv }- \text { cinv }), \quad \operatorname{lmak}+\mathrm{bInv}+(\operatorname{inv}-\operatorname{cinv}), \quad \text { cinvLSB }+(\text { inv }- \text { cinv }) .
$$

Theorem 1.9 provides also an alternative proof of the following combinatorial interpretations for $q$ Stirling numbers of the second kind, where the first two interpretations were proved by Ksavrelof and Zeng [4] and the third interpretation was first proved by Stanton (see [12]).

Corollary 1.12

$$
S_{q}(n, k)=\sum_{\pi \in \mathcal{P}_{n}^{k}} q^{\operatorname{mak} \pi}=\sum_{\pi \in \mathcal{P}_{n}^{k}} q^{\operatorname{lmak} \pi}=\sum_{\pi \in \mathcal{P}_{n}^{k}} q^{\mathrm{lsb} \pi+\left(\begin{array}{l}
\mathrm{k} \\
2
\end{array}\right)} .
$$

Indeed, an (unordered) partition can be identified with an ordered partition without inversion, i.e.,

$$
\mathcal{P}_{n}^{k}=\left\{\pi \in \mathcal{O} \mathcal{P}_{n}^{k} \mid \operatorname{inv} \pi=0\right\}
$$

Since the statistic bInv vanishes on (unordered) partitions and the identity cinvLSB $=1 \mathrm{sb}+2\left(\begin{array}{c}\mathrm{k} \\ 2\end{array}\right)$ holds on $\mathcal{P}^{k}$, it is then trivial to obtain Corollary 1.12

There are three main ingredients in the proof of Theorem 1.9 a bijection which maps ordered partitions to walks on some digraphs, the transfer-matrix method which permits to compute the generating function of walks using determinants, and the evaluation of determinants.

\section{Ordered partitions and walks in digraphs}

\subsection{Encoding ordered partitions by walks}

Let $\pi=B_{1}-B_{2}-\cdots-B_{k}$ in $\mathcal{O P}_{n}^{k}$ and $i$ an integer in $[n]$. The restriction $B_{j}(\leq i):=B_{j} \cap[i]$ of the block $B_{j}$ is said to be active if $B_{j} \nsubseteq[i]$ and $B_{j} \cap[i] \neq \emptyset$, complete if $B_{j} \subseteq[i]$. We define the restriction of $\pi$ on $[i]$, called the $i$-th trace of $\pi$, by

$$
T_{i}(\pi)=B_{1}(\leq i)-B_{2}(\leq i)-\cdots-B_{k}(\leq i)
$$

with the empty restrictions being omitted. The sequence $\left(T_{i}(\pi)\right)_{1 \leq i \leq n}$ is called the trace of the partition $\pi$. We denote by act $i \pi$ and $\operatorname{com}_{i} \pi$ the numbers of active blocks and complete blocks, respectively, in $T_{i}(\pi)$ and set

$$
\omega_{i}(\pi)=\left(\operatorname{com}_{i} \pi, \operatorname{act}_{i} \pi\right) \quad \text { for } \quad 1 \leq i \leq n,
$$

with $\omega_{0}(\pi)=(0,0)$. The sequence $\left(\omega_{i}(\pi)\right)_{0 \leq i \leq n}$ is called the walk of the partition $\pi$.

For instance, if $\pi=\{6\}-\{3,5,7\}-\{1,4,10\}-\{9\}-\{2,8\}$, then $T_{6}(\pi)=\{6\}-\{3,5, \cdots\}-\{1,4, \cdots\}-$ $\{2, \cdots\}$, where each active block ends with an ellipsis, and we get $\omega_{6}(\pi)=(1,3)$.

Definition 2.1 Let $D=(V, E)$ be the digraph on $V=\mathbb{N}^{2}$ with edges set $E$ defined by

$$
E=\left\{(u, v) \in V^{2} \mid u=v=(x, y) \text { with } y>0 \quad \text { or } \quad u-v=(0,1),(1,0),(1,-1)\right\}
$$

Clearly there are four types of edges. An edge $(u, v)$ of $D$ is called:

- North if $v=u+(0,1)$;

- East if $v=u+(1,0)$;

- South-East if $v=u+(1,-1)$;

- Null if $v=u$.

For any integer $k \geq 0$, let $V_{k}=\{(i, j) \in V \mid i+j \leq k\}$ and $D_{k}$ be the restriction of the digraph $D$ on $V_{k}$. An illustration of $D_{k}$ is given in Figure 1 . 


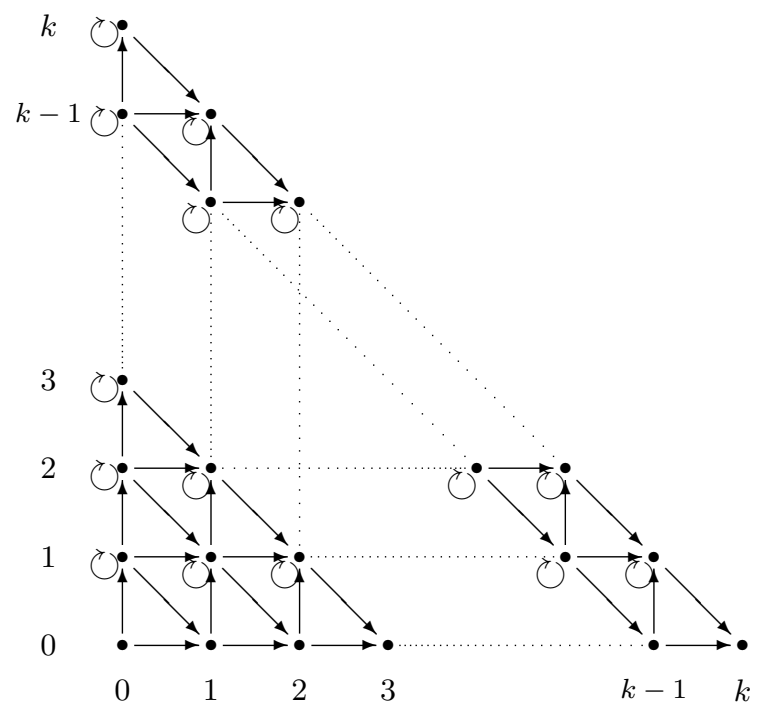

Fig. 1: The digraph $D_{k}$

Definition 2.2 A walk of depth $k$ and length $n$ in $D$ is a sequence $\omega=\left(v_{0}, v_{1}, \ldots, v_{n}\right)$ of points in $V$ such that $v_{0}=(0,0), v_{n}=(k, 0)$ and $\left(v_{i}, v_{i+1}\right)$ is an edge of $D$ for $i=0, \ldots, n-1$. Moreover, the abscissa and height of the step $\left(v_{i}, v_{i+1}\right)$ are the abscissa and ordinate of $v_{i}$ respectively. Let $\Omega_{n}^{k}$ be the set of walks of depth $k$ and length $n$ and $\Omega^{k}$ be the set of walks of depth $k$ in $D$.

The following result characterizes the walks of ordered partitions.

Proposition 2.3 For $n \geq k \geq 0$, the walks of the partitions in $\mathcal{O} \mathcal{P}_{n}^{k}$ are exactly the walks in $\Omega_{n}^{k}$.

We need a refinement of the notion of walk.

Definition $2.4 \mathrm{~A}$ walk diagram of depth $k$ and length $n$ is a pair $(\omega, \xi)$, where $\omega=\left(v_{0} v_{1} \cdots v_{n}\right)$ is a walk in $\Omega_{n}^{k}$ and $\xi=\left(\xi_{i}\right)_{1 \leq i \leq n}$ is a sequence of integers such that $1 \leq \xi_{i} \leq q$ if the $i$-th step is (Null or South-East) of height $q$, and $1 \leq \xi_{i} \leq p+q+1$ if the $i$-th step is (North or East) of abscissa pand height $q$.

Denote by $\Delta_{n}^{k}$ the set of path diagrams of depth $k$ and length $n$. The main ingredient of our proof is a mapping from $\Delta_{n}^{k}$ to $\mathcal{O} \mathcal{P}_{n}^{k}$ which keeps track of several statistics of Steingrímsson.

Theorem 2.5 There exists a bijection $\psi: \Delta_{n}^{k} \rightarrow \mathcal{O P}_{n}^{k}$ such that if $h=(\omega, \xi)$ is in $\Delta_{n}^{k}$ and if the $i$-th step of $w$ is of abscissa $p$ and height $q$, and of type:

(i) North or East : then, $i \in(\mathcal{O} \cup \mathcal{S})(\psi(h))$,

$(\mathrm{lcs}+\mathrm{rcs})_{\mathrm{i}}(\psi(\mathrm{h}))=\mathrm{q}$ and $(\mathrm{lsb}+\mathrm{rsb})_{\mathrm{i}}(\psi(\mathrm{h}))=\mathrm{p}$,

$\operatorname{los}_{\mathrm{i}}(\psi(\mathrm{h}))=\xi_{\mathrm{i}}-1$ and $\operatorname{ros}_{\mathrm{i}}(\psi(\mathrm{h}))=\mathrm{p}+\mathrm{q}+1-\xi_{\mathrm{i}}$.

(ii) South-East or Null : then, $i \in(\mathcal{T} \cup \mathcal{C})(\psi(h))$,

$(\mathrm{lcs}+\mathrm{rcs})_{\mathrm{i}}(\psi(\mathrm{h}))=\mathrm{p} \quad$ and $\quad(\mathrm{lsb}+\mathrm{rsb})_{\mathrm{i}}(\psi(\mathrm{h}))=\mathrm{q}-1$,

$\operatorname{lsb}_{\mathrm{i}}(\psi(\mathrm{h}))=\xi_{\mathrm{i}}-1 \quad$ and $\quad \operatorname{rsb}_{\mathrm{i}}(\psi(\mathrm{h}))=\mathrm{q}-\xi_{\mathrm{i}}$

\subsection{Generating functions of walks}

For $0 \leq k \leq n$, let $\mathbf{t}=\left(t_{1}, t_{2}, t_{3}, t_{4}, t_{5}, t_{6}, t_{7}\right)$ and

$$
\begin{aligned}
Q_{n, k}(\mathbf{t}):= & \sum_{\pi \in \mathcal{O} \mathcal{P}_{n}^{k}} t_{1}^{(\mathrm{lcs}+\mathrm{rcs})(\mathcal{O} \cup \mathcal{S}) \pi} t_{2}^{(\mathrm{lcs}+\mathrm{rcs})(\mathcal{T} \cup \mathcal{C}) \pi} t_{3}^{\operatorname{rsb}(\mathcal{T} \cup \mathcal{C}) \pi} \\
& \times t_{4}^{\operatorname{lsb}(\mathcal{T} \cup \mathcal{C}) \pi} t_{5}^{\operatorname{ros}(\mathcal{O} \cup \mathcal{S}) \pi} t_{6}^{\operatorname{los}(\mathcal{O} \cup \mathcal{S}) \pi} t_{7}^{(\mathrm{lsb}+\mathrm{rsb})(\mathcal{O} \cup \mathcal{S}) \pi} .
\end{aligned}
$$


Given a walk $\omega$, define the weight $\mathrm{v}(\omega)$ of $\omega$ to be the product of the weights of all its steps, where the weight of a step of abscissa $i$ and height $j$ is:

$$
\begin{cases}t_{1}^{i} t_{7}^{j}[i+j+1]_{t_{5}, t_{6}} & \text { if the step is North or East; } \\ t_{2}^{i}[j]_{t_{3}, t_{4}} & \text { if the step is Null or South-East. }\end{cases}
$$

It follows easily from Theorem 2.5 that

$$
\sum_{\omega \in \Omega_{n}^{k}} \mathrm{v}(\omega)=Q_{n, k}(\mathbf{t})
$$

Denote by $|\omega|$ the length of the walk $\omega$. Then, using the above identity, we get

$$
Q_{k}(a ; \mathbf{t}):=\sum_{n \geq 0} Q_{n, k}(\mathbf{t}) a^{n}=\sum_{w \in \Omega^{k}} \mathrm{v}(w) a^{|\omega|} .
$$

It is obvious that the number of vertices of $D_{k}$ is equal to

$$
\widehat{k}:=1+2+\cdots+(k+1)=\frac{(k+1)(k+2)}{2} .
$$

Let $v_{1}, \cdots, v_{\widehat{k}}$ be the vertices of $D_{k}$ arranged according to the following order: $(i, j) \leq\left(i^{\prime}, j^{\prime}\right)$ if and only if $i+j<i^{\prime}+j^{\prime}$ or $\left(i+j=i^{\prime}+j^{\prime}\right.$ and $\left.j \geq j^{\prime}\right)$. For instance, we get $v_{1}=(0,0), v_{2}=(0,1), v_{3}=$ $(1,0), v_{4}=(0,2), v_{5}=(1,1), v_{6}=(2,0), \cdots, v_{\widehat{k}}=(k, 0)$.

The adjacency matrix $A_{k}$ of $D_{k}$ relative to the valuation $\mathrm{v}$ is the $\widehat{k} \times \widehat{k}$ matrix defined by

$$
A_{k}(i, j)= \begin{cases}\mathrm{v}\left(v_{i}, v_{j}\right) & \text { if }\left(v_{i}, v_{j}\right) \text { is an edge of } D_{k} \\ 0 & \text { otherwise. }\end{cases}
$$

Applying the transfer-matrix method (see e.g. [9], Theorem 4.7.2]), we obtain

$$
Q_{k}(a ; \mathbf{t})=\frac{(-1)^{1+\widehat{k}} \operatorname{det}\left(I-a A_{k} ; \widehat{k}, 1\right)}{\operatorname{det}\left(I-a A_{k}\right)}
$$

where $(B ; i, j)$ denotes the matrix obtained by removing the $i$-th row and $j$-th column of $B$ and $I$ is the $\widehat{k} \times \widehat{k}$ identity matrix.

In order to prove Theorem 1.9 it suffices to evaluate the following specializations of (14):

$$
\begin{aligned}
f_{k}(a ; x, y, t, u) & =Q_{k}(a ; x, x, x, y, t, u, y), \\
g_{k}(a ; z, t, u) & =Q_{k}(a ; 1, z, 1, z, t, u, 1) .
\end{aligned}
$$

Let $A_{k}^{\prime}$ and $A_{k}^{\prime \prime}$ be the adjacency matrix of $D_{k}$ relative to the weight function $\mathrm{v}^{\prime}$ and $\mathrm{v}^{\prime \prime}$ obtained from the weight function $\mathrm{v}$ by making the substitution (15) and $(16)$, respectively. Namely, the weights $\mathrm{v}^{\prime}(e)$ and $\mathrm{v}^{\prime \prime}(e)$ of an edge $e=\left((i, j),\left(i^{\prime}, j^{\prime}\right)\right)$ of $D_{k}$ with initial vertex $(i, j)$ are :

$$
\mathbf{v}^{\prime}(e)= \begin{cases}x^{i} y^{j}[i+j+1]_{t, u} & \text { if }\left(i^{\prime}, j^{\prime}\right)=(i, j+1) \text { or }(i+1, j) \\ x^{i}[j]_{x, y} & \text { if }\left(i^{\prime}, j^{\prime}\right)=(i, j) \text { or }(i+1, j-1)\end{cases}
$$

and

$$
\mathbf{v}^{\prime \prime}(e)= \begin{cases}{[i+j+1]_{t, u}} & \text { if }\left(i^{\prime}, j^{\prime}\right)=(i, j+1) \text { or }(i+1, j) \\ z^{i}[j]_{z} & \text { if }\left(i^{\prime}, j^{\prime}\right)=(i, j) \text { or }(i+1, j-1) .\end{cases}
$$

Now, for each $k \geq 0$ let

$$
M_{k}=I-a A_{k}^{\prime} \quad \text { and } \quad N_{k}=I-a A_{k}^{\prime \prime} .
$$

Then by (14, , 15) and (16) we have

$$
\begin{aligned}
f_{k}(a ; x, y, t, u) & =\frac{(-1)^{1+\widehat{k}} \operatorname{det}\left(M_{k} ; \widehat{k}, 1\right)}{\operatorname{det} M_{k}}, \\
g_{k}(a ; z, t, u) & =\frac{(-1)^{1+\widehat{k}} \operatorname{det}\left(N_{k} ; \widehat{k}, 1\right)}{\operatorname{det} N_{k}}
\end{aligned}
$$


for each $k \geq 1$.

Since $M_{n}$ and $N_{n}$ are upper triangular matrices it is easy to see that for each $n \geq 1$

$$
\begin{aligned}
& \operatorname{det} M_{n}=\prod_{m=1}^{n} \prod_{i=0}^{m}\left(1-a x^{i}[m-i]_{x, y}\right), \\
& \operatorname{det} N_{n}=\prod_{m=1}^{n} \prod_{k=0}^{n-m}\left(1-a z^{k}[m]_{q}\right) .
\end{aligned}
$$

The evaluation of $\operatorname{det}\left(M_{n} ; \widehat{n}, 1\right)$ and $\operatorname{det}\left(N_{n} ; \widehat{n}, 1\right)$ is highly non trivial.

Theorem 2.6 Let $n \geq 1$ be a positive integer. Then

$$
\operatorname{det}\left(M_{n} ; \widehat{n}, 1\right)=(-1)^{\left(\begin{array}{c}
n \\
2
\end{array}\right)} a^{n} x^{\left(\begin{array}{c}
n \\
2
\end{array}\right)}[n]_{t, u} ! \prod_{m=1}^{n-1} \prod_{i=1}^{m}\left(1-a x^{i}[m-i+1]_{x, y}\right),
$$

Theorem 2.7 Let $n \geq 1$ be a positive integer. Then

$$
\operatorname{det}\left(N_{n} ; \widehat{n}, 1\right)=(-1)^{\left(\begin{array}{c}
n \\
2
\end{array}\right)} a^{n}[n]_{t, u} ! \prod_{m=1}^{n-1} \prod_{k=1}^{n-m}\left(1-a z^{k-1}[m]_{z}\right) .
$$

It is now trivial to obtain the following result.

Corollary 2.8 For $k \geq 0$, we have

$$
\begin{aligned}
f_{k}(a ; x, y, t, u) & =\frac{a^{k} x^{\left(\begin{array}{c}
k \\
2
\end{array}\right)[k]_{t, u} !}}{\prod_{i=1}^{k}\left(1-a[i]_{x, y}\right)}, \\
g_{k}(a ; z, t, u) & =\frac{a^{k}[k]_{t, u} !}{\prod_{i=1}^{k}\left(1-a z^{k-i}[i]_{z}\right)} .
\end{aligned}
$$

Finally Theorem 1.9 follows immediately from Corollary 2.8 and the following lemma.

Lemma 2.9 The following identities hold:

$$
\begin{aligned}
\phi_{k}(a ; x, y, t, u) & =f_{k}\left(a ; x, y, x y t, u y^{2}\right), \\
\varphi_{k}(a ; z, t, u) & =g_{k}\left(a z^{k-1} ; 1 / z, t, u / z\right) .
\end{aligned}
$$

Therefore in order to prove Theorem 1.9 it remains to prove Theorems 2.6 and 2.7

\section{Determinantal computations}

\subsection{Proof of Theorem 2.6}

The matrix $M_{n}$ can be defined recursively by

$$
M_{0}=(1) \quad \text { and } \quad M_{n}=\left(\frac{M_{n-1} \mid \bar{M}_{n-1}}{O_{n+1, \widehat{n-1}} \mid \widehat{M}_{n-1}}\right) \quad \text { for } n \geq 1,
$$

where $\widehat{M}_{n-1}$ is the $(n+1) \times(n+1)$ matrix

$$
\widehat{M}_{n-1}=\left(\delta_{i j}-a x^{i-1}[n+1-i]_{x, y}\left(\delta_{i j}+\delta_{i+1, j}\right)\right)_{1 \leq i, j \leq n+1}
$$

and $\bar{M}_{n-1}$ is the $\widehat{n-1} \times(n+1)$ matrix

$$
\bar{M}_{n-1}=\left(\frac{O_{\widehat{n-2}, n+1}}{\check{M}_{n-1}}\right)
$$


with the $n \times(n+1)$ matrix

$$
\check{M}_{n-1}=\left(-a x^{i-1} y^{n-i}[n]_{t, u}\left(\delta_{i j}+\delta_{i+1, j}\right)\right)_{1 \leq i \leq n, 1 \leq j \leq n+1} .
$$

Here $\delta_{i j}$ stands for the Kronecker delta and $O_{m, n}$ denotes the $m \times n$ zero matrix. Let

$$
\mathrm{K}_{n}=\widehat{n}-1=\frac{n(n+3)}{2},
$$

and let $P_{n}=\left(M_{n} ; \widehat{n}, 1\right)$, i.e the $\mathrm{K}_{n} \times \mathrm{K}_{n}$ matrix obtained from $M_{n}$ by deleting the $\widehat{n}$ th row and the first column. The problem is to compute $\operatorname{det} P_{n}$ for $n \geq 1$. The matrix $P_{n}$ can also be defined recursively:

$$
P_{n}=\left(\frac{P_{n-1} \mid \bar{P}_{n-1}}{X_{n-1} \mid \widehat{P}_{n-1}}\right)
$$

Here $\bar{P}_{n-1}$ is a $\mathrm{K}_{n-1} \times(n+1)$ matrix, $X_{n-1}$ is a $(n+1) \times \mathrm{K}_{n-1}$ matrix, and $\widehat{P}_{n-1}$ is a $(n+1) \times(n+1)$ matrix. The idea here is to use the following well-known formula for any block matrix with an invertible square matrix $A$,

$$
\operatorname{det}\left(\frac{A \mid B}{C \mid D}\right)=\operatorname{det} A \cdot \operatorname{det}\left(D-C A^{-1} B\right) .
$$

Since the entries of $C A^{-1} B$ are also written by minors, we guess these entries and prove it by induction.

\subsection{Proof of Theorem 2.7}

Let $F=\left\{F_{n}\right\}_{n=1}^{\infty}$ be a sequence of non-zero functions in finitely many variables $v_{1}, v_{2}, \ldots$ We use the convention that $F_{n} !=\prod_{k=1}^{n} F_{k}$ and

$$
\left[\begin{array}{l}
n \\
k
\end{array}\right]_{F}= \begin{cases}\frac{F_{n} !}{F_{k} ! F_{n-k} !}, & \text { if } 0 \leq k \leq n \\
0, & \text { otherwise }\end{cases}
$$

We prove Theorem 2.7 22) by considering the following matrix $N_{n}(x, a)$, which generalize the matrix $N_{n}$ (set $x=1$ and $F_{n}=[n]_{t, u}$ to obtain $N_{n}$ ). Let $N_{n}(x, a)$ be the matrix defined inductively by:

$$
N_{0}(x, a)=(x)
$$

and

$$
N_{n}(x, a)=\left(\begin{array}{c|c}
N_{n-1}(x, a) \mid \bar{N}_{n-1}(x, a) \\
\hline O_{n+1, \widehat{n-1}} \mid \widehat{N}_{n-1}(x, a)
\end{array}\right)
$$

where $\widehat{N}_{n-1}(x, a)$ is the $(n+1) \times(n+1)$ matrix defined by

$$
\widehat{N}_{n-1}(x, a)=\left(x \delta_{i j}-a q^{i-1}[n+1-i]_{q}\left(\delta_{i j}+\delta_{i+1, j}\right)\right)_{1 \leq i, j \leq n+1}
$$

and $\bar{N}_{n-1}(x, a)$ is the $\widehat{n-1} \times(n+1)$ matrix

$$
\left(\frac{O_{\widehat{n-2}, n+1}}{\check{N}_{n-1}}\right)
$$

with the $n \times(n+1)$ matrix

$$
\check{N}_{n-1}=\left(-a F_{n} \cdot\left(\delta_{i j}+\delta_{i+1, j}\right)\right)_{1 \leq i \leq n, 1 \leq j \leq n+1} .
$$

Let $\dot{N}_{n}(x, a)$ denote the matrix obtained from $N_{n}(x, a)$ by deleting the $\widehat{n}$ th row and the first column. Then the following theorem is sufficient to prove our result. 
Theorem 3.1 We have

$$
\operatorname{det} \dot{N}_{n}(x, a)=(-1)^{\frac{n(n-1)}{2}} a^{n} F_{n} ! x^{n} \prod_{m=1}^{n-1} \prod_{k=1}^{n-m}\left(x-a q^{k-1}[m]_{q}\right) .
$$

Setting $x=1$ and $F_{n}=[n]_{t, u}$ we obtain Theorem 2.7 .

Here our strategy is as follows. We regard $\operatorname{det} N_{n}(x, a)$ as a polynomial in $x$ and find all linear factors. Finally we check the leading coefficient in the both sides.

\section{References}

[1] Carlitz (L.), q-Bernoulli and Eulerian numbers, Trans. Amer. Math. Soc., 76 (1954), 332-350.

[2] Carlitz (L.), A combinatorial property of q-Eulerian numbers, Amer. Math. Monthly, 82 (1975), 51-54. 332-350.

[3] Foata (D.) and Zeilberger (D.), Denert's permutation statistic is indeed Euler-Mahonian, Studies in Appl. Math., 83 (1990), 31-59.

[4] Ksavrelof (G.) and Zeng (J.), Nouvelles statistiques de partitions pour les q-nombres de Stirling de seconde espèce, Discrete Math., Vol. 256, Issue 3, 2002, 743-758.

[5] Milne (S.), Restricted growth functions, rank row matching of partition lattices, and q-Stirling numbers, Adv. Math., 43 (1982), 173-196.

[6] Sagan (B.), A maj statistics for set partitions, European J. Combin., 12 (1991), 69-79.

[7] Simion (S.) and Stanton (D.), Specializations of generalized Laguerre polynomials, SIAM J. MATH. ANAL., 25 (1994), 712-719.

[8] Simion (S.) and Stanton (D.), Octabasic Laguerre polynomials and permutation statistics, J. of Computational and Applied Math. 68 (1996), 297-329.

[9] R. P. Stanley, Enumerative combinatorics I, Cambridge Studies in Advanced Mathematics 49, 1997.

[10] Steingrímsson (E.), Statistics on ordered partitions of sets, preprint, 1999.

[11] Wachs (M.), $\sigma$-Restricted Growth Functions and p,q-stirling numbers, J. Combin. Theory Ser. A, 68 (1994), 470-480.

[12] Wachs (M.) and White (D.), p, q-Stirling numbers and set partition statistics, J. Combin. Theory Ser. A, 56 (1991), 27-46.

[13] White (D.), Interpolating Set Partition Statistics, J. Combin. Theory Ser. A, 68 (1994), 262-295.

[14] Viennot (X.), Une théorie combinatoire des polynômes orthogonaux généraux, Notes de conférences à l'UQAM, Montréal, 1983.

[15] Zeng (J.) and Zhang (C. G.), A q-analog of Newton's series, Stirling functions and Eulerian functions, Results in Math., 25 (1994), 370-391. 